\title{
Chemical biology at the Broad Institute
}

The Broad Institute was founded with the vision of creating a truly collaborative biomedical research environment, and small molecules are a central focus.

"Collaborative at its core" is how Stuart Schreiber characterizes research at the Broad Institute. In 2004, Eric Lander, a genome biologist, David Altschuler, a human geneticist, Todd Golub, a cancer biologist, and Schreiber, a chemical biologist, founded the Broad Institute (http://www. broad.mit.edu) based on a shared vision for a new way to approach genome-based biomedical research. "We like to get together as a community," Schreiber says, "and think about the greatest opportunities for impacting science and society. The plans that result may be very high risk, but we're willing to pursue them if a successful outcome would be transforming."

Basic biomedical research at the Broad Institute is directed towards genome biology and cell circuits, medical and population genetics, computational biology and bioinformatics, and chemical biology. Diseases with a significant need for new therapeutics - cancer, psychiatric diseases, metabolic diseases and infectious diseases-are the other major research direction. The Broad Institute includes six core faculty members, whose laboratories are based at the institute. Cross-disciplinary research is encouraged by a laboratory design in which chemists and biologists are fully integrated, often occupying adjacent benches.

In keeping with the integration of chemistry and biology, synthetic chemistry and high-throughput screening provide critical small-molecule research tools. With this fundamental reliance on identifying smallmolecule modulators, chemical biologists at the institute are rethinking every aspect of high-throughput screening with the goal of creating a more rapid and robust process. An optimal approach to small-molecule discovery, as Schreiber envisions it, involves three key steps: "get the right chemistry, let the system reveal itself, and systematically identify targets."

"The chemistry that's yielded drug-like compounds creates compounds with a very high $s p^{2}$ content," Schreiber mentions. "In more colloquial terms this translates mentally into compounds that are flat." In contrast, chemistry at the Broad Institute is increasingly focused on diversityoriented synthesis (DOS). "We use chemistry that gives rise to the types of interesting compounds typical of natural products with features such as ring systems, stereogenic centers and heteroatoms," describes Damian Young, a chemistry group leader. "We're opening ourselves up to all of chemical space."

Following the identification of a screening hit, a large medicinal chemistry effort is typically required for lead optimization. The structurally complex DOS compounds being developed at the Broad Institute are created with the idea of expediting optimization. "We try to restrict ourselves to three to five chemical steps," Schreiber says, and the compounds are created by modular syntheses in which, "for example, the four quadrants of the skeleton come together in one or two steps, so you can easily go back and change them." Further, each of the four pieces has multiple orthogonal chemical handles for readily generating diversity by adding appendages. In Schreiber's view, it only makes sense to develop a screening library that "anticipates all the needs of chemistry at each step along the process."

Unlike the in vitro screens typically used in the pharmaceutical industry, most screening at the institute involves cell-based, phenotypic screens. As Schreiber describes, the power of this approach is that it "uses the biology to probe the chemistry." The main challenge of phenotypic screening is the next step-identifying the small-molecule target. An anecdote from Schreiber's laboratory illustrates just how difficult a challenge this is: "Roughly speaking we've published papers describing 20 such targetidentification projects. The good news is that it worked 20 times; the very disconcerting news is that that is over a 20 -year period." A current goal at the Broad Institute is to develop methods for systematic target identification. By combining mass spectrometry-based proteomics with DOS libraries that can readily be modified with chemical handles, researchers at the Broad are making progress toward this goal. However, as Schreiber notes, "Do we have the systematic target-identification problem solved? No way."

All screening data from the Broad Institute is made freely available in ChemBank (http://chembank.broad.harvard.edu/), which, because the assays are all run against the same collection of compounds, as Schreiber notes, "provides an unbelievable level of annotation." Paul Clemons, a group leader in computational chemical biology, is analyzing this data to investigate "the propensities of natural products versus DOS mole-

"We like to get together
as a community,"
Schreiber says,
"and think about the
greatest opportunities
for impacting science
and society."

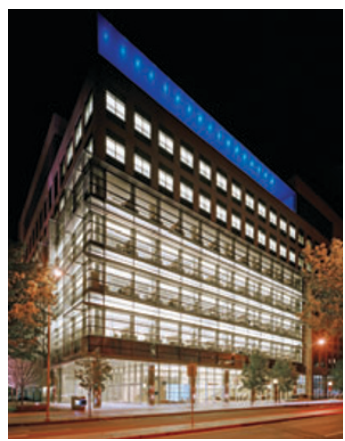

cules versus drug-like compounds to score in different screening assays." Although he realizes that people have strong feelings on this subject, Clemons hopes that "quantifying these outcomes in an unbiased analysis will put the conversation on a more scientific footing."

Identifying new therapeutic leads is one application of the chemical biology small-molecule discovery effort. Bridget Wagner, a group leader in chemical biology, is hoping to use small molecules to induce insulin production in vivo. Most attempts to address type I diabetes have involved inducing cells to produce insulin in vitro and then transplanting these cells back into the pancreas. "If one uses small molecules to tweak the transcriptional activities or the chromatin state of beta cells or other pancreatic cells, could they be coaxed to produce insulin?"Wagner asks. "If we find a compound that has some effect, it would be huge because type I diabetes is still only treated with insulin injection."

"Antibiotics tend to defy classical drug-like properties," notes Deborah Hung, a Broad faculty member. Antibiotics typically need to pass the bacterial cell wall; however, little is known about the chemical properties that govern bacterial cell permeability. Hung is using mass spectrometry-based approaches to define "which chemicals can get in, and which cannot." Hung also emphasizes the need to move beyond "the classical antibiotic approach of screening rapidly growing bacteria looking for death" to look more closely at virulence.

"The beauty of the Broad," Hung says, "is the ability to truly integrate multidisciplinary approaches." In describing his shift from heading a classic academic laboratory at Harvard to pursuing research in the collaborative environment of the Broad, Schreiber says, "I feel like a graduate student again-I can't wait to come to work every day."

Joanne Kotz, Cambridge, Massachusetts 